Abstract 0C-119 Table 1 Relative quantity of inflammatory genes from SDD patients pre and post treatment with Placebo (P) or Mesalazine (M) (Median [inter-quartile range])

\begin{tabular}{|c|c|c|c|c|c|c|}
\hline \multirow{2}{*}{$\begin{array}{l}\text { Median (IOR) } \\
\text { Genes }\end{array}$} & \multicolumn{2}{|l|}{$P$ group } & \multirow[b]{2}{*}{ p Value } & \multicolumn{2}{|l|}{ M group } & \multirow[b]{2}{*}{ p Value } \\
\hline & Pre & Post & & Pre & Post & \\
\hline NOD2 & $0.8938(0.76-1.57)$ & $1.021(0.60-1.55)$ & 0.6632 & $1.359(0.79-1.96)$ & $0.5925(0.40-0.94)$ & 0.0002 \\
\hline PAR2 & $1.841(1.40-2.34)$ & $1.571(1.20-2.08)$ & 0.4080 & $1.944(1.49-3.37)$ & $1.056(0.77-1.25)$ & 0.0007 \\
\hline TNF $\alpha$ & $0.6611(0.47-1.05)$ & $0.6341(0.34-1.15)$ & 0.2977 & $0.9170(0.61-1.40)$ & $0.4435(0.36-0.69)$ & 0.0034 \\
\hline IL1B & $0.7416(0.47-1.40)$ & $1.092(0.37-1.49)$ & 0.2575 & $0.9633(0.37-1.96)$ & $0.6220(0.37-1.01)$ & 0.0024 \\
\hline TLR4 & $1.377(0.94-2.49)$ & $1.465(1.05-1.94)$ & 0.3604 & $1.537(1.01-1.96)$ & $0.8932(0.70-1.52)$ & 0.0479 \\
\hline TLR9 & $0.4506(0.34-0.73)$ & $0.5264(0.30-0.68)$ & 0.4488 & $0.7991(0.39-1.17)$ & $0.4976(0.32-0.64)$ & 0.0215 \\
\hline MYD88 & $1.765(1.51-2.66)$ & $1.678(1.22-2.44)$ & 0.6013 & $1.780(1.15-2.99)$ & $1.134(0.90-1.76)$ & 0.0105 \\
\hline
\end{tabular}

Methods Patients with confirmed SDD underwent an unprepared flexible sigmoidoscopy and biopsies at baseline and after 12 weeks treatment, completing diaries of pain and bowel habit. They were randomised to receive $3 \mathrm{~g}$ per day of mesalazine $(\mathrm{M})$ or identical placebo (P) for 12 weeks with follow-up visits at $2 \& 4$ weeks. RNA from sigmoid biopsies was analysed using a custom made gene card. Gene expression and changes in symptoms were assessed between baseline and final visits using Wilcoxon signed-rank test.

Results 43 volunteers were recruited (F:M; 24:19), but 11 withdrew during the study resulting in 18 and 14 participants in the $\mathrm{P}$ and $\mathrm{M}$ groups respectively. $M$ significantly reduced important inflammatory and pain genes including those involved in response to bacterial ligands (Abstract OC-119 table 1), changes not seen with P. M but not $\mathrm{P}$ significantly reduced the duration of abdominal pain (Median (range) (hrs/day) P group Pre 1.0 (0-5), Post 0.65 (0-4) p0.1919; M Group Pre 3.0 (0-20), Post 0.125 (0-5.5) p0.0413).

Conclusion This pilot study suggests that mesalazine significantly alters many of the pathways mediating immune activation by bacteria thought to contribute to pain in SDD and may provide benefit for patients. However further larger studies are required to confirm these mechanisms and efficacy of mesalazine in this group.

Competing interests $\mathrm{J}$ Smith grant/research support from: Wellcome Trust Training Fellowship, Conflict with: $£ 17000$ Gift from Dr Falk Pharma for manufacture of medication for the study, D Humes: None declared, K Garsed: None declared, C Lam: None declared, A Zaitoun: None declared, A Bennett: None declared, J Scholefield: None declared, R Spiller: None declared.

\section{Bapen symposium}

\section{OC-120 THE "NUTRITION SUPPORT PYRAMID"-COMPOSITION AND TRENDS IN A REGIONAL PAEDIATRIC COHORT FROM SOUTH EAST SCOTLAND}

\section{doi:10.1136/gutjnl-2012-302514a.120}

${ }^{1} \mathrm{C}$ E Paxton, ${ }^{2} \mathrm{P}$ Henderson, ${ }^{2} \mathrm{D} \mathrm{C}$ Wilson. ${ }^{1}$ Paediatric Gastroenterology and Nutrition, NHS Lothian, UK; ${ }^{2}$ Child Life and Health, University of Edinburgh, Edinburgh, UK

Introduction Within nutrition support (NS) there is a spectrum of complexity ranging from oral supplements via enteral tube feeding (ETF) to parenteral nutrition (PN) for intestinal failure (IF). Nutrition support teams (NST) are involved with those children with chronic or complex nutrition needs on home ETF (HETF) and home $\mathrm{PN}$ (HPN). There is no current robust data available on the prevalence of children on each level of what we newly describe as the nutrition support pyramid (NSP).

Aims To introduce the concept of the NSP and to describe the composition and temporal trends in the NSP in a UK regional paediatric cohort.

Methods We performed a retrospective cohort study (database/ clinical note review) of all children $(<16 \mathrm{yrs})$ in SE Scotland requiring NS over a 7-year period (2004-2010). We divided all children having NS into levels of the NSP, which comprises four levels of nutrition support; a 5th level is children with IF who have required transplantation $(\mathrm{Tx})$ to achieve intestinal adaptation. The NSP base comprises children receiving HETF, the second level is children with severe upper GI dysmotility requiring jejunal HETF, the penultimate level is children with type II IF (prolonged hospital $\mathrm{PN}$ ), and the top level is type III IF (children who receive HPN). Poisson regression models and Fisher's exact testing were then used to compare the period prevalence (per calendar year) of children $<16$ yrs requiring NS and the proportions of each level of the NSP between the two epochs of 2004-2006 and 2008-2010.

Results There were a total of 780 NS episodes in 702 children $(51 \%$ male); 69 (10\%) had multiple episodes of NS. Median (IOR) age at commencing NS was $1.0(0.2-4.7)$ yrs. There was a significant increase in the period prevalence of children requiring NS between the two epochs $(p=0.004)$. However, although the number of children requiring HETF (level 1) rose from 372 to 422 between the two epochs, there was a non-significant change in the shape of the NSP (determined by the relative size of each level) between the two epochs ( $p=0.736$ ). During the entire study period a total of 715 NS episodes were located on the base level (HETF); 31 on the second level (jejunal HETF); 21 on the penultimate level (type II IF); and 14 on the top level (type III IF), with four children requiring Tx to achieve enteral autonomy.

Conclusion We have introduced the concept of a NSP, not previously described, and shown that the NSP has significantly increased in size (number of children requiring NS) without a significant change in the shape (relative distribution of complexity). The NSP is a simple tool which allows us to show a significant increase in NST workload without any decrease in complexity over just 7 years.

Competing interests None declared.

\section{Free papers AUGIS oesophago-gastric OC-121 FIBROBLAST ACTIVATION IN THE TUMOUR MICROENVIRONMENT PROMOTES TUMOUR CELL INVASION AND RESISTANCE TO CHEMOTHERAPY IN OESOPHAGEAL ADENOCARCINOMA}

doi:10.1136/gutjnl-2012-302514a.121

A L Hayden, M F Derouet, F Noble, J N Primrose, J P Blaydes, G Thomas, T J Underwood. * Cancer Sciences, University of Southampton, Southampton, UK

Introduction Stromal and other non-malignant cells have the potential to undergo modifications that can synergistically create a supportive microenvironment for tumour growth, invasion and metastasis. Oesophageal adenocarcinoma (EAC) is characterised by early invasion, leading to metastatic disease and therefore only $20 \%$ of patients are suitable for treatment with curative intent. Cancer associated fibroblasts (CAFs) have an activated, myofibroblastic phenotype and have been recognised as mediators of tumour 
progression in a range of solid tumours. This study investigates the role of CAFs in EAC invasion and resistance to chemotherapy.

Methods Functional biological analyses comparing primary fibroblasts from tumour stroma (CAF) and normal oesophagus (NOF) were carried out using organotypic culture, transwell invasion assays, collagen-1 gel contraction assays, siRNA gene silencing and colony forming assays. T-Tests (>95\% CI) were carried out for all statistical analyses.

Results Primary oesophageal CAFs displayed an activated phenotype as demonstrated by $\alpha$-SMA expression and increased collagen- 1 gel contraction in comparison to NOFs $(p<0.01)$. CAF conditioned medium supported tumour colony formation in the presence of cisplatin and 5-Fluorouracil compared to NOF conditioned medium $(p<0.05)$. Ex vivo analysis revealed a twofold $(p<0.05)$ increase in EAC cell invasion in response to primary (CAF) conditioned medium in transwell invasion assays that was replicated in $3 \mathrm{D}$ organotypic models containing co-cultures of fibroblasts and EAC tumour cells. Down-regulation of the CAF secreted molecule Periostin (PN) resulted in a $70 \%$ reduction in tumour cell invasion in transwell assays $(p<0.05)$, and a total loss of invasion in organotypic culture. Furthermore, collagen- 1 gel contraction was abrogated by PN down-regulation. NOFs exposed to TGF- $\beta$ from $72 \mathrm{~h}$ demonstrate features of myofibroblastic activation including, PN expression and the ability to support increased EAC tumour cell invasion $(\mathrm{p}<0.05)$.

Conclusion This study has demonstrated that oesophageal derived primary CAF protect EAC cells from chemotherapy and promote tumour cell invasion. Increased $\boldsymbol{\alpha}$-SMA expression and collagen- 1 gel contraction indicates CAF have a myofibroblast like phenotype. PN siRNA reduced gel contraction supports a hypothesis of autocrine regulation of the myofibroblast phenotype. Therefore targeting pathways that determine fibroblast activation may offer a novel therapy for preventing oesophageal cancer invasion and metastasis.

Competing interests None declared.

\section{OC-122 ENHANCED RECOVERY AFTER UPPER GASTROINTESTINAL SURGERY (ERAUGIS) IMPROVES OUTCOMES IN UPPER GASTROINTESTINAL (UGI) CANCER}

doi:10.1136/gutjnl-2012-302514a.122

A J Beamish,* D S Y Chan, T D Reid, R Barlow, I Howell, G Blackshaw, G Clark, W G Lewis. Upper GI Surgery, University Hospital of Wales, Cardiff, UK

Introduction The enhanced recovery after surgery (ERAS) principles of optimising nutrition, minimising the surgical stress response and early mobilisation have been reported to improve outcomes in colorectal surgery, but data in UGI surgery is thin. The aim of this study was to determine the influence on outcomes of an ERAS programme in UGI cancer surgery.

Methods Two hundred and eight consecutive patients, 89 managed in the ERAUGIS programme and 119 historical controls (CON) were studied in this prospective observational cohort study, and the primary outcome measures were hospital length of stay (LoS) and 30-day morbidity (classified according to the Clavien-Dindo (CD) criteria).

Results No significant differences in age $(p=0.938)$, gender $(p=0.518)$, tumour site $(p=0.461)$, radiological stage $(p=0.219)$ or histopathological stage $(p=0.116)$ were identified between the two cohorts. ERAUGIS was associated with significant reductions in: overall LoS (median 12 vs 15 days, $\mathrm{p}=0.001$ ), critical care LoS (median 1 vs 2 days, $\mathrm{p}<0.0001$ ) and critical care related cancellations $(2.2 \%$ vs $8.4 \%, p=0.004)$. No significant differences in readmission rate (ERAUGIS vs CON $=11 \%$ vs $5 \%, p=0.134$ ) or CD morbidity class $(p=0.089)$ were observed. Multivariable analysis revealed that
ERAUGIS $(p<0.0001)$ and anatomical site of surgery (thoracic vs abdominal, $\mathrm{p}<0.0001)$ were independently and significantly associated with hospital LoS.

Conclusion ERAUGIS was associated with a fourfold reduction in CC cancellation rates, halved CC LoS and reduced total LoS by $20 \%$ without increasing serious operative morbidity. These findings show that ERAS principles outlined in colorectal surgery can be modified and applied safely to patients diagnosed with UGI cancer with significant patient safety, quality and economic benefits.

Abstract OC-122 Table 1 Multivariable analysis of factors affecting hospital length of stay

\begin{tabular}{llll}
\hline Variable & HR & $\mathbf{9 5 \%}$ CI & p Value \\
\hline ERAUGIS & 0.382 & 0.226 to 0.645 & $<0.0001$ \\
Site of surgery & 2.459 & 1.515 to 3.992 & $<0.0001$ \\
\hline
\end{tabular}

Competing interests None declared.

\section{OC-123 OBESITY DRIVES RADIORESISTANCE AND ENHANCES GENOMIC INSTABILITY IN OESOPHAGEAL ADENOCARCINOMA}

doi:10.1136/gutjnl-2012-302514a.123

A M Mongan,* N Lynam-Lennon, S Maher, G Pidgeon, J O'Sullivan, J Reynolds. Department of Surgery, Institute of Molecular Medicine, Dublin, Ireland

Introduction Obesity is strongly associated with oesophageal adenocarcinoma (OAC). However, its role in regulating radiosensitivity and genomic instability is unknown. We developed an isogenic model of radioresistance in OAC called OE33R. We assessed levels of anaphase bridges, a functional genomic instability event, in OE33R compared to an age and passage matched control (OE33P). Spindle assembly checkpoint (SAC) control genes involved in regulating genomic instability were analysed in both cell lines following exposure to adipose conditioned media (ACM). Radiosensitivity following ACM treatment was investigated in OE33R and OE33P cell lines. The aim of this study was to characterise the isogenic radioresistant OAC cell model in terms of genomic instability, SAC gene expression and survival in response to adipose conditioned media (ACM) cultured from obese and nonobese OAC patients.

Methods OE33R and OE33P cell lines were cultured with ACM from obese and nonobese patients. We assessed anaphase bridges in both lines and quantified the number of bridges present over the total cell number. Expression of five SAC genes (MAD2L2, BUB1B, CDC20, CENPE, and ESPL1) was assessed using qPCR. Survival was determined in both cells lines following ACM treatment using a clonogenic assay.

Results OE33P and OE33P showed a significant increase in anaphase bridges in response to ACM ( $p<0.05, p<0.001$ respectively). This increase in anaphase bridge formation was three times greater in the resistant line $(p<0.05)$. Levels of anaphase bridge formation correlated with obesity status (BMI and VFA, $\mathrm{p}<0.05$ ). OE33R cells treated with ACM showed significantly increased expression of the SAC genes MAD2L2 and BUB1B compared to controls $(p<0.01)$. Expression of MAD2L2 and BUB1B correlated with obesity status $(p<0.05)$. OE33P cells treated with ACM showed increased radioresistance $(p<0.05)$. In contrast, in the resistant OE33R cell line, ACM treatment reversed this radioresistance $(p<0.001)$.

Conclusion Obesity drives genomic instability and alterations in SAC gene expression in radioresistant $\mathrm{OAC}$ and alters radiosensitivity in OAC.

Competing interests None declared. 